\title{
Influência da polifarmácia e do uso de medicamentos inapropriados para idosos sobre a taxa de filtração glomerular
}

\author{
Influence of polypharmacy and use of inappropriate medication for the elderly on the glomerular
} filtration rate

Influencia de la polifarmacia y el uso de medicación inapropiada en ancianos sobre la tasa de filtración glomerular

Recebido: 24/03/2021 | Revisado: 30/03/2021 | Aceito: 02/04/2021 | Publicado: 04/04/2021

\author{
Diego Bruno Morais \\ ORCID: https://orcid.org/0000-0002-8310-1813 \\ Universidade Federal de Juiz de Fora, Brasil \\ E-mail: moraisdiegobruno@gmail.com \\ João Pedro Vasconcelos Paolinelli \\ ORCID: https://orcid.org/0000-0001-7981-6763 \\ Universidade Federal de São João del - Rei, Brasil \\ E-mail: joaopedro.paolinelli@gmail.com \\ Soraia de Freitas Tavares Dâmaso \\ ORCID: https://orcid.org/0000-0002-0756-180X \\ Universidade José do Rosário Vellano, Brasil \\ E-mail: sftdamaso@gmail.com \\ André Oliveira Baldoni \\ ORCID: https://orcid.org/0000-0001-6379-0415 \\ Universidade Federal de São João del - Rei, Brasil \\ E-mail: andrebaldoni@ufsj.edu.br \\ Alba Otoni \\ ORCID: https://orcid.org/0000-0002-8126-3026 \\ Universidade Federal de São João del - Rei, Brasil \\ E-mail: albaotoni@ufsj.edu.br
}

\begin{abstract}
Resumo
Objetivo: Investigar a associação entre a alteração da Taxa de Filtração Glomerular estimada em pessoas idosas com o uso de Medicamentos Potencialmente Inapropriados para idosos e polifarmácia. Metodologia: Estudo transversal e analítico desenvolvido a partir de dados de 1216 idosos usuários do Sistema Público de Saúde que apresentavam exames de Taxa de Filtração Glomerular estimada, sendo a mesma considerada alterada quando $<60 \mathrm{~mL} / \mathrm{min} / 1,73 \mathrm{~m}^{2}$. Foram realizadas análises descritivas, análise univariada "teste de qui-quadrado $\left(\chi^{2}\right)$ ou exato de Fisher" e as variáveis que apresentaram $\mathrm{p}<0,2$ foram incluídas na análise de regressão logística multivariada. Resultados: Do total de pacientes, 541 (44,5\%) apresentavam Taxa de Filtração Glomerular estimada alterada, 943 (77,5\%) utilizavam polifarmácia e 588 (48,3\%) usavam Medicamentos Potencialmente Inapropriados para idosos. A análise multivariada mostrou que homens acima de 80 anos apresentam maiores chances de terem a Taxa de Filtração Glomerular estimada alterada e que a prática da polifarmácia aumentou em 1,62 as chances de alterações neste parâmetro $(\mathrm{p}=0,004 ; O R: 1,62 ; \mathrm{IC}: 1,67$ - 2,24). Além disso, a utilização de Medicamentos Potencialmente Inapropriados aumentou em 1,49 vezes as chances de ter a Taxa de Filtração Glomerular estimada alterada ( $\mathrm{p}=0,003$ OR: 1,49; IC:1,15 - 1,93). Conclusão: Este estudo evidenciou uma alta frequência de uso de polifarmácia e Medicamentos Potencialmente Inapropriados em idosos e essa população se mostrou com maior chance de comprometimento das funções renais. Assim, este achado traz um alerta à saúde pública e aponta para a necessidade de se investir em uma abordagem terapêutica como foco na segurança do paciente idoso.

Palavras-chave: Taxa de filtração glomerular; Polimedicação; Medicamentos potencialmente inapropriados; Idosos.
\end{abstract}

\begin{abstract}
Objective: To investigate the association between the change in the estimated Glomerular Filtration Rate in elderly people with the use of Potentially Inappropriate Medicines for the elderly and polypharmacy. Methodology: Crosssectional and analytical study based on data from 1216 elderly users of the Public Health System who had estimated Glomerular Filtration Rate exams, the same being considered altered when $<60 \mathrm{~mL} / \mathrm{min} / 1.73 \mathrm{~m} 2$. Descriptive analyzes, univariate analysis "chi-square test $\left(\chi^{2}\right)$ or Fisher's exact test" were performed and variables with $p<0.2$ were included in the multivariate logistic regression analysis. Results: Of the total number of patients, $541(44.5 \%)$ had altered estimated Glomerular Filtration Rate, 943 (77.5\%) used polypharmacy and 588 (48.3\%) used Potentially Inappropriate Medicines for the elderly. Multivariate analysis showed that men over 80 years of age are more likely to have their
\end{abstract}


estimated Glomerular Filtration Rate altered and that the practice of polypharmacy increased the chances of alterations in this parameter by 1.62 ( $\mathrm{p}=0.004$; OR: 1.62; CI: 1, $67-2.24)$. In addition, the use of Potentially Inappropriate Medicines increased the chances of having the estimated Glomerular Filtration Rate altered by 1.49 times $(p=0.003$ OR: 1.49; CI: 1.15 - 1.93). Conclusion: This study showed a high frequency of using of polypharmacy and Potentially Inappropriate Medicines in the elderly and this population was shown to have a greater chance of impairing renal functions. Thus, this finding brings an alert to public health and points to the necessity to invest in a therapeutic approach as a focus on the safety of elderly patients.

Keywords: Glomerular filtration rate; Polypharmacy; Potentially inappropriate medication; Aged.

\section{Resumen}

Objetivo: Investigar la asociación entre el cambio en la Tasa de Filtración Glomerular estimada en ancianos con el uso de Medicamentos Potencialmente Inapropiados para ancianos y la polifarmacia. Metodología: Estudio transversal y analítico a partir de datos de 1216 ancianos usuarios del Sistema Público de Salud a los que se les realizó una prueba de Tasa de Filtración Glomerular estimada, considerándose alterada la misma cuando $<60 \mathrm{~mL} / \mathrm{min} / 1,73 \mathrm{~m} 2$. Se realizaron análisis descriptivos, análisis univariante "prueba de chi-cuadrado $\left(\chi^{2}\right)$ o prueba exacta de Fisher" y las variables con $p$ $<0,2$ se incluyeron en el análisis de regresión logística multivariante. Resultados: Del total de pacientes, 541 (44,5\%) tenían Tasa de Filtración Glomerular estimada alterada, 943 (77,5\%) utilizaban polifarmacia y 588 (48,3\%) utilizaban Medicamentos Potencialmente Inapropiados para ancianos. El análisis multivariado mostró que los hombres mayores de 80 años tienen más probabilidades de tener una Tasa de Filtración Glomerular estimada alterada y que la práctica de la polifarmacia aumenta las posibilidades de alteraciones en este parámetro en 1,62 ( $\mathrm{p}=0,004$; OR: 1,62; IC: 1, 67 2,24). Además, el uso de Medicamentos Potencialmente Inapropiados aumentó las posibilidades de haber alterado la Tasa de Filtración Glomerular estimada en 1,49 veces ( $\mathrm{p}=0,003$ OR: 1,49; IC: 1,15 - 1,93). Conclusión: Este estudio mostró una alta frecuencia de uso de polifarmacia y Medicamentos Potencialmente Inapropiados en ancianos y esta población demostró tener una mayor probabilidad de alterar las funciones renales. Así, este hallazgo trae una alerta a la salud pública y apunta a la necesidad de invertir en un enfoque terapéutico como foco en la seguridad de los pacientes ancianos.

Palabras clave: Tasa de filtration glomerular; Polifarmacia; Lista de medicamentos potencialmente inapropiados; Ancianos.

\section{Introdução}

É crescente o número de idosos em todo o mundo e a World Health Organization (WHO) estima que 22\% da população mundial será representada por pessoas com 60 anos ou mais, em 2050 (Organização Pan-Americana de Saúde [OPAS], 2018; World Health Organization [WHO], 2016). Atualmente, no Brasil, baseado no fato de que 14\% da população é idosa (Fundação Getúlio Vargas [FGV], 2020) e vem numa linha ascendente de crescimento, houve adequações e aumento de políticas públicas de saúde voltadas às pessoas nessa faixa etária, bem como maior utilização de tecnologias em saúde, como o uso amplo de medicamentos, a fim de melhorar a sobrevida e qualidade de vida dos idosos (Brasil, 2014).

Apesar desses avanços para aprimoramento da assistência a pessoa idosa, o envelhecimento é inexorável e à medida que a idade avança, o organismo envelhece de forma simultânea em todos os sistemas, ocasionando alterações fisiológicas relacionadas ao desgaste natural do corpo (American Geriatrics Society [AGS], 2019; Shi \& Klotz, 2011; Stager et al., 2019). Associadas a esse contexto, as comorbidades são frequentes, além da exposição a fatores de risco ao longo da vida, aumentando de forma exponencial a suscetibilidade a complicações dos quadros de saúde e morte (AGS, 2019).

Diante desse processo natural do envelhecimento, é importante compreender que independente do avançar da idade, existem fatores que podem favorecer ou, ao contrário, prejudicar a fisiologia dessa faixa etária, a depender da possibilidade de modificação dos mesmos. Aqueles fatores que são intrínsecos e individuais são imutáveis como, por exemplo, a genômica e a anatomia de cada pessoa. Já os extrínsecos, como ambiente no qual a pessoa idosa está inserida, o estilo de vida, e os fatores mais contemporâneos, como o uso de polifarmácia e de Medicamentos Potencialmente Inapropriados (MPI), podem ser ajustados de forma a minimizar o impacto negativo na vida do idoso (AGS, 2019; Nascimento et al., 2017; Shi \& Klotz, 2011; Stader et al., 2019).

Em especial, no tocante a mudança nos hábitos de vida das pessoas em qualquer faixa etária, há que se considerar que não é fácil e envolve a participação ativa da própria pessoa, do núcleo familiar e da equipe de saúde. Entre os fatores modificáveis, 
o uso da polifarmácia e de MPI são fatores que, em um primeiro momento, dependem da ação direta da equipe de saúde e são passíveis de modificações e ajustes que adequem a rotina medicamentosa do idoso sem trazer maiores riscos para o mesmo (Baldoni et al., 2014; Rocha et al., 2020). Porém, mesmo diante das consolidadas recomendações da literatura sobre a parcimônia ao expor o paciente à polifarmácia e a cautela com a utilização de MPI em idosos, ainda é comum na prática clínica se identificar pessoas na faixa etária idosa em uso indiscriminado de polifarmácia e de MPI (Nascimento et al., 2017; Rocha et al., 2020).

Tendo em vista que grande parte dos medicamentos possuem excreção renal, e entre as alterações sistêmicas consequentes ao envelhecimento encontra-se a redução das funções renais, torna-se imprescindível a avaliação prévia da saúde dos rins antes da associação de novos medicamentos às prescrições de pessoas idosas (Lassiter et al., 2013). A fim de melhorar o atendimento aos pacientes com as melhores evidências, a International Society of Nephrology (ISN) através do Kidney Disease: Improving Global Outcomes (KDIGO - $3^{\circ}$ Volume) apresenta os exames que podem ser utilizados avaliar a função renal, sendo eles: relação albumina/creatinina, creatinina sérica, e a taxa de filtração glomerular (TFG) entre outros (International Society of Nephrology [ISN], 2013; KDIGO).

Nem sempre existe a preocupação com a avaliação das funções renais frente ao uso de polifarmácia e MPI em pessoas idosas nas unidades de saúde brasileiras. Além disso, na literatura internacional, existem evidências de associação da polifarmácia e MPI com a alteração da TFG para além do fisiológico em idosos, contudo, não é do nosso conhecimento que existam estudos em populações brasileiras com esse objetivo (Secora et al., 2019). Tendo em vista a tendência crescente do aumento da população idosa e a redução das funções renais, além da necessidade de reconhecimento do perfil de idosos assistidos pelo Sistema Único de Saúde (SUS) no Brasil, o objetivo desse estudo foi investigar a associação entre a alteração da Taxa de Filtração Glomerular estimada (TFGe) em pessoas idosas com o uso de MPI para idosos e polifarmácia.

\section{Metodologia}

Trata-se de um estudo transversal analítico, desenvolvido em município do centro-oeste mineiro/Brasil. Foi aprovado pelo Comitê de Ética em Pesquisa da Universidade Federal de São João del-Rei (UFSJ), parecer número 1.686.007 CAAE 58481416.3.0000.5545 e seguiu as recomendações do Strengthening the Reporting of Observational Studies in Epidemiology Statement (STROBE).

A população elegível foi determinada a partir dos seguintes critérios: todos os idosos (60 anos ou mais) acompanhados na Atenção Primária de Saúde (APS) que receberam a dispensação de medicamentos, pelo menos uma vez no ano de 2015 em uma das cinco farmácias do Sistema Único de Saúde (SUS) em uma cidade do centro-oeste mineiro/Brasil e que tivessem registrado, no prontuário, pelo menos um valor de creatinina sérica, para estimativa da TFGe. Como critérios de exclusão foi considerado: pacientes cujos prontuários continham informações incompletas e pacientes com diagnóstico prévio de doença renal crônica terminal.

As variáveis explicativas foram coletadas a partir do prontuário eletrônico, dentre elas: as características sociodemográficas: sexo, idade, estado civil, além da utilização de polifarmácia (prescrição de cinco ou mais medicamentos) e a utilização de MPI de acordo com os Critérios de Beers (AGS, 2019; Rocha et al., 2020). Esses critérios correspondem à lista mais citada e utilizada como referência de MPI para idosos (AGS, 2019). No Brasil, o consenso brasileiro de medicamentos potencialmente inapropriados para idosos também referencia esta informação (Oliveira et al., 2016).

Para identificação da variável resposta, TFGe alterada ou não, os valores de creatinina sérica disponíveis no período do estudo foram coletados dos prontuários para cálculo da TFGe por meio da equação Chronic Kidney Disease Epidemiogy Collaboration (CKD-EPI) e os resultados foram categorizados em TFGe alterada ( $\left.<60 \mathrm{ml} / \mathrm{min} / 1,73 \mathrm{~m}^{2}\right)$ e TFGe não alterada ( $\geq 60 \mathrm{ml} / \mathrm{min} / 1,73 \mathrm{~m}^{2}$ ), sendo essa a variável desfecho do estudo. O estágio da Doença Renal Crônica (DRC) foi classificado em categorias (ml/min/1,73m²): G1 (>89), G2 (60-89), G3a (45-60), G3b (30-44), G4 (15-29) e G5 (<15) de acordo com os critérios 
do KDIGO (ISN, 2013). As análises estatísticas foram realizadas pelo programa Statistical Package for the Social Sciences Version 19 (SPSS).

Para caracterização do perfil sociodemográfico da população estudada foi realizada análise descritiva. Para realizar as análises univariada e multivariada, a idade foi agrupada em 60 a 79 anos e acima de 80, e a situação conjugal foi categorizada a partir da classificação padrão com e sem companheiro. Para as variáveis categóricas foram feitas tabelas de distribuição de frequências e para as variáveis contínuas utilizou-se medidas de tendência central e variabilidade média, desvio padrão e mediana e intervalos interquartis conforme a distribuição de normalidade avaliada pelo teste de normalidade Kolmogorov-Smirnov. A análise univariada foi realizada utilizando o "teste qui-quadrado $\left(\chi^{2}\right)$ ou exato de Fisher" e as variáveis que apresentaram $p<0,2$ foram incluídas na análise de regressão logística multivariada.

\section{Resultados}

Entre os 2511 pacientes idosos que receberam medicamentos em uma das cinco farmácias do município, foram incluídos no estudo 1216 que preenchiam os critérios de inclusão. A maioria fazia uso da polifarmácia e aproximadamente 50\% estava em uso de MPI (Tabela 1). Entre os 541 pacientes com TFGe alterada, 443 (81,9\%) foram classificados no estágio de doença renal G3A, 89 (16,5\%) no G3B e $9(1,6 \%)$ no G4, sendo que destes 306 estavam em uso de MPI.

Tabela 1. Variáveis sociodemográficas, presença de polifarmácia e uso de MPI associadas à TFGe alterada em pacientes idosos de um município do centro-oeste mineiro em 2015 (n=1216).

\begin{tabular}{|c|c|c|c|}
\hline & \multicolumn{2}{|c|}{ TFGe*** } & \multirow[b]{2}{*}{$\begin{array}{c}\text { Valor - } \mathbf{p}^{*} \\
\text { OR [IC 95\%] }\end{array}$} \\
\hline & $\begin{array}{l}\text { Não alterada } \\
\quad(n=675)\end{array}$ & $\begin{array}{c}\text { Alterada } \\
(n=541)\end{array}$ & \\
\hline \multirow[t]{4}{*}{ Sexo } & & & $<0,001$ \\
\hline & $429(50,8)$ & $416(49,2)$ & 1,00 \\
\hline & $246(66,3)$ & $125(33,7)$ & $1,91[1,5-2,5]$ \\
\hline & & & $<0,001$ \\
\hline \multicolumn{4}{|l|}{ Idade } \\
\hline $60-79$ & $652(57,5)$ & $482(42,5)$ & 1,00 \\
\hline$\geq 80$ & $23(28,1)$ & $59(71,9)$ & $2,78[2,3-3,4]$ \\
\hline & & & 0,028 \\
\hline \multicolumn{4}{|l|}{ Estado Civil } \\
\hline Com Companheiro & $186(46,4)$ & $215(53,6)$ & 1,00 \\
\hline Sem Companheiro & $340(57,9)$ & $187(31,9)$ & $1,17[1,0-1,4]$ \\
\hline & & & $<0,001$ \\
\hline \multicolumn{4}{|l|}{ Uso de Polifarmácia } \\
\hline 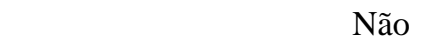 & $192(70,3)$ & $81(29,7)$ & 1,00 \\
\hline Sim & $483(51,2)$ & $460(48,8)$ & $2,26[1,7-3,0]$ \\
\hline \multirow[t]{3}{*}{ Uso de MPI**** } & & & $<0,001$ \\
\hline & $394(62,6)$ & $235(37,4)$ & 1,00 \\
\hline & $281(47,9)$ & $306(52,1)$ & $1,83[1,5-2,3]$ \\
\hline
\end{tabular}

*Teste $\mathrm{X}^{2}$ ou exato de Fisher; **TFGe: Taxa de filtração glomerular estimada; ***MPI: Medicamentos potencialmente inapropriados. Fonte Autores (2021).

Estes pacientes utilizavam 8025 medicamentos, sendo que 742 (9,3\%) são considerados MPI. Registrou-se maior consumo de medicamentos que atuam no sistema nervoso central (Tabela 2). 
Tabela 2. Medicamentos Potencialmente Inapropriados utilizados pelos pacientes idosos da cidade do centro-oeste mineiro em $2015(n=742)$.

\begin{tabular}{cc}
\hline MPI* & $\mathbf{N}(\mathbf{\%})$ \\
\hline Clonazepam & $229(30,9)$ \\
Diazepam & $149(20,1)$ \\
Amitriptilina & $137(18,5)$ \\
Nifedipina & $51(6,9)$ \\
Alprazolam & $46(6,2)$ \\
Nitrofurantoina & $23(3,1)$ \\
Imipramina & $22(3,0)$ \\
Nortriptilina & $22(3,0)$ \\
Amiodarona & $21(2,8)$ \\
Fenobarbital & $18(2,4)$ \\
Digoxina & $10(1,3)$ \\
Flurazepam & $7(0,9)$ \\
Metildopa & $7(0,9)$ \\
Total & $742(100)$ \\
\hline
\end{tabular}

*Medicamentos Potencialmente Inapropriados. Fonte Autores (2021).

No modelo final as variáveis sexo, idade, uso de polifarmácia e uso de MPI mostraram associação independente e significativa com a TFGe alterada. Os homens mais velhos, submetidos à prática da polifarmácia, bem como utilizando MPI tiveram maiores chances de terem a TFGe alterada sendo os Odds Ratio 1,87, 2,85, 1,62 e 1,48, respectivamente (Tabela 3).

Tabela 3. Análise multivariada das variáveis que apresentaram p-Valor $<0,2$ ou que tem impacto conhecido, pela literatura, na TFGe em pacientes idosos da cidade do centro-oeste mineiro em $2015(\mathrm{n}=1216)$.

Valor $-\mathbf{p}^{*} \quad$ OR * [IC 95\%]

Sexo
Masculino
$<0,001$
$1,87[1,4-2,5]$

Idade
$>80$
$<0,001$
$2,85[2,3-3,5]$

\section{Estado Civil}

Sem Companheiro

0,490

$1,06[0,9-1,2]$

Polifarmácia

0,004

$1,62[1,2-2,2]$

Uso de MPI**

0,003

$1,48[1,1-1,9]$

*Regressão Logística; **Medicamentos Potencialmente Inapropriados. Fonte Autores (2021).

\section{Discussão}

Um dos maiores desafios da saúde pública é promover o uso seguro e racional de medicamentos, pois a prática da automedicação, presença de polifarmácia e prescrição de MPI ainda são prevalentes, sobretudo em idosos (Arrabal \& Salvi, 2018; Rocha, 2020). Os resultados obtidos no presente estudo reforçam a necessidade de uma atenção especial na população geriátrica, pois a idade avançada (>80 anos), a polifarmácia e a utilização de MPI se mostraram significativamente associados à alteração na TFGe. 
Os dados referentes especificamente à polifarmácia chamam atenção, pois há um considerável aumento na chance de alteração da TFGe em idosos (OR: 1,62). Resultado semelhante ao estudo de Araújo et al. (2019), que evidenciaram maiores chances de se ter as funções renais comprometidas com polifarmácia em pacientes acima de 65 anos (OR: 4,04). Além disso, os autores mostraram uma correlação positiva entre a redução da função renal e a polifarmácia reforçando a necessidade de investigação prévia da função renal antes de implementar medicamentos de forma simultânea (König et al., 2017). Sugere-se uma reflexão sobre a possibilidade de desprescrever medicamentos, principalmente aqueles de potencial nefrotóxico, como os anti-inflamatórios não esteroidais (AINEs), pois há evidências de melhora significativa na função renal diante dessa conduta (Palacios et al., 2015). Os AINEs são anti-inflamatórios que agem inibindo as enzimas cicloxigenases e suprimindo a síntese de prostaglandinas (PGs). Como a homeostase renal depende da ação das PGs, o mecanismo da maioria dos medicamentos desta classe pode induzir a diminuição da perfusão renal total, provocando redução da TFG, vasoconstrição renal, isquemia medular, podendo, em certas ocasiões, resultar em doença renal aguda, nefrite intersticial, síndrome nefrótica e necrose papilar renal (Silva et al., 2014; Ungprasert et al., 2015). Sendo assim, a longitudinalidade do cuidado, incluindo o acompanhamento farmacoterapêutico, associado a uma análise contínua dos parâmetros clínicos, principalmente da TFGe, é de fundamental importância para se evitar possíveis complicações causadas pela polifarmácia, que também estão relacionadas ao uso de MPI

Além dos possíveis comprometimentos à saúde do paciente a partir do uso da polifarmácia, Baldoni et al. (2014), alertam que essa prática é um dos principais fatores associados ao uso de MPI. Estes medicamentos trazem mais riscos do que benefícios ao tratamento, e seu uso é considerado preditor de mortalidade em idosos (Nascimento et al., 2017). Com foco no uso de MPI, os resultados do estudo atual evidenciaram que os idosos que usavam os MPI apresentaram maior chance de possuírem TFGe alterada (OR: 1,48). Esses achados vão ao encontro de outros estudos que evidenciaram associação significativa entre o uso de MPI e alteração da TFGe e que, consequentemente, aumentam significativamente o número de relatos de eventos adversos na população idosa (Araújo et al., 2019; Palacios et al., 2015; Musso et al., 2015). Considerando os achados persistentes da literatura sobre a relação "nociva" do uso de MPI e comprometimento das funções renais, além dos consagrados Critérios de Beers (AGS, 2019) como principal referência de uso inapropriado e agrupados de acordo a classificação Anatomic Therapeutic Chemical (ATC) (WHO, 2020), pode-se inferir que, aparentemente, ainda existem prescrições dos MPI não balizadas nos parâmetros recomendados ou a prática do "off-label" dos medicamentos, sendo de responsabilidade do prescritor os riscos e benefícios que o mesmo possa causar.

Dos MPI específicos utilizados por esta população destacam-se os benzodiazepínicos. Esta classe merece atenção, principalmente na faixa etária estudada, pois podem aumentar o risco de queda e fratura, e com a excreção reduzida devido à injuria renal, estes efeitos podem ser exacerbados (AGS, 2019; Donnelly et al., 2017). Ressalta-se que para a prescrição ou desprescrição destes medicamentos é muito importante individualizar e seguir os protocolos (AGS, 2019; Bourgeois et al., 2014; Reeve et al., 2017;), definir a conduta terapêutica conforme o estado clínico atual, avaliar a resposta à redução das doses, a evolução e a relação benefício/risco deve tender à melhora na qualidade de vida. Aos demais medicamentos o destaque se dá ao aumento do risco de sedação, hipotensão ortostática e isquemia miocárdica (AGS, 2019; Secora et al., 2019).

O presente estudo revelou um maior número de participantes do sexo feminino. Embora os homens sejam mais afetados pela DRC, provavelmente justificado pelos efeitos hormonais e hábitos de vida menos saudáveis, as mulheres, no Brasil, são culturalmente mais assíduas nas unidades de saúde e, por isso, eventualmente são diagnosticadas com maior frequência em estágios iniciais e, consequentemente, impactam em um melhor prognóstico e no aumento da prevalência destas no sexo feminino (Botton, Cúnico, \& Strey, 2017; Carrero et al., 2018; Malta et al., 2017). Mostra-se então, a importância de medidas que aumentem a participação masculina nos ambientes de saúde, como campanhas focadas na população masculina e busca ativa, visando uma abordagem preventiva para a maioria das doenças, em especial, nas crônicas não transmissíveis. 
Por fim, o presente estudo apresenta algumas limitações, dentre as quais destaca-se que, pelo delineamento transversal, não é possível estabelecer a relação de causa e efeito. Mesmo assim, os resultados gerais desse estudo são relevantes para a prática clínica, pois contribuem para reflexões acerca de benefícios e prejuízos da terapia medicamentosa na população geriátrica. Essas reflexões merecem atenção por parte não só dos profissionais prescritores, como também da equipe assistencial de pessoas idosas, no sentido de que toda a abordagem terapêutica deve ser baseada na efetividade terapêutica e segurança do paciente, evitando MPI e tendo cautela na prática da polifarmácia, sendo esta utilizada somente quando for considerada indispensável para o equilíbrio orgânico dos envolvidos.

\section{Conclusão}

A frequência de pessoas idosas em uso de polifarmácia e de MPI é elevada e essas pessoas se mostraram com maior chance de comprometimento das funções renais. Esse achado traz um alerta à saúde pública quanto a necessidade de se investir em uma abordagem terapêutica com foco na segurança ao paciente idoso. É preciso que estimule a utilização de parâmetros laboratoriais e clínicos individualizados para a prescrição adaptada a cada indivíduo. Esse formato de abordagem terapêutica pode reduzir os riscos de eventos adversos, possíveis evoluções negativas da DRC e, consequentemente, reduzir os gastos para o Sistema de Saúde.

\section{Agradecimentos}

Os autores agradecem à Universidade Federal de São João del-Rei (UFSJ), à secretaria municipal de saúde de Divinópolis/MG e ao Conselho Nacional de Desenvolvimento Científico e Tecnológico pelo incentivo e financiamento da pesquisa.

\section{Referências}

American Geriatrics Society (AGS). (2019). Updated AGS Beers Criteria® for Potentially Inappropriate Medication use in older adults. Journal of the American Geriatrics Society, 67(4), 674-694. https://doi.org/10.1111/jgs.15767

Araújo, L. U., Santos, D. F., Bodevan, E. C., Cruz, H. L. da, Souza, J. de, \& Silva-Barcellos, N. M. (2019). Patient safety in primary health care and polypharmacy: cross-sectional survey among patients with chronic diseases. Revista Latino-Americana de Enfermagem, 27, e3217. https://doi.org/10.1590/15188345.3123 .3217

Arrabal Júnior, J. M., \& Salvi, J. D. O. (2018). Fatores associados à automedicação em uma farmácia comunitária de Ouro Preto Do Oeste, Rondônia. Acta Biomédica Brasiliensia, 9(2), 107. https://doi.org/10.18571/acbm.177

Baldoni, A. D. O., Ayres, L. R., Martinez, E. Z., Dewulf, N. D. L. S., Dos Santos, V., \& Pereira, L. R. L. (2014). Factors associated with potentially inappropriate medications use by the elderly according to Beers criteria 2003 and 2012. International Journal of Clinical Pharmacy, 36(2), 316-324. https://doi.org/10.1007/s11096-013-9880-y

Botton, A., Cúnico, S. D. \& Strey, M. N. (2017). Diferenças de gênero no acesso aos serviços de saúde: problematizações necessárias. Revista Mudança Psicologia e saúde, 25(1), 67 - 72. https://www.metodista.br/revistas/revistas-metodista/index.php/MUD/article/view/7009/5608

Bourgeois, J., Elseviers, M. M., Bortel, L. V., Petrovic, M. \& Stichele, R. H. V. (2014). Feasibility of discontinuing chronic benzodiazepine use in nursing home residents: a pilot study. European Journal of Clinical Pharmacology, 70, 1251-1260. DOI 10.1007/s00228-014-1725-7

Brasil. (2014). Ministério da Saúde. Secretaria de Ciência, Tecnologia e Insumos Estratégicos. Departamento de Assistência Farmacêutica e Insumos Estratégicos. Cuidado Farmacêutico na Atenção Básica - Caderno 2: Capacitação para implantação dos serviços de clínica farmacêutica. ISBN 978-85-3342198-1. Brasília, DF. http://bvsms.saude.gov.br/bvs/publicacoes/cuidado_farmaceutico_atencao_basica_saude_2.pdf

Carrero, J. J., Hecking, M., Chesnaye, N. C., \& Jager, K. J. (2018). Sex and gender disparities in the epidemiology and outcomes of chronic kidney disease. Nature Reviews Nephrology, 14(3), 151-164. https://doi.org/10.1038/nrneph.2017.181

do Nascimento, M. M. G., Mambrini, J. V. de M., Lima-Costa, M. F., Firmo, J. O. A., Peixoto, S. W. V., \& de Loyola Filho, A. I. (2017). Potentially inappropriate medications: predictor for mortality in a cohort of community-dwelling older adults. European Journal of Clinical Pharmacology, 73(5), 615-621. https://doi.org/10.1007/s00228-017-2202-x

Donnelly, K., Bracchi, R., Hewitt, J., Routlede, B. C. (2017). Benzodiazepines, Z-drugs and the risc of hip fracture: A systematic review and meta-analysis. Plos One,12(4), e0174730. https://doi.org/10.1371/journal.pone.0174730 
Fundação Getúlio Vargas (FGV). (2020). Social - Centro de Políticas Sociais. Mapas - Idosos. https://cps.fgv.br/mapas-idosos-elderly-maps

International Society of Nephrology (ISN). (2013). Kidney Internacional Suplements. Kidney Disease: Improving Global Outcomes (KDIGO) 2013. Clinical Practice Guideline for the Evaluation and Management of Chronic Kidney Disease. Official Journal of the International Society of Nephrology, 3, 1-150. https://kdigo.org/wp-content/uploads/2017/02/KDIGO_2012_CKD_GL.pdf

König, M., Gollasch, M., Demuth, I., \& Steinhagen-Thiessen, E. (2017). Prevalence of Impaired Kidney Function in the German Elderly: Results from the Berlin Aging Study II (BASE-II). Gerontology, 63(3), 201-209. https://doi.org/10.1159/000454831

Lassiter, J., Bennett, W. M., \& Olyaei, A. J. (2013). Drug Dosing in Elderly Patients with Chronic Kidney Disease. Clinics in Geriatric Medicine, 29(3), 657705. https://doi.org/10.1016/j.cger.2013.05.008

Malta, D. C., Bernal, R. T. I., Lima, M. G., de Araújo, S. S. C., da Silva, M. M. A., Freitas, M. I. de F., \& Barros, M. B. de A. (2017). Noncommunicable diseases and the use of health services: Analysis of the National Health Survey in Brazil. Revista de Saúde Pública, 51, 1S-10S. https://doi.org/10.1590/S15188787.2017051000090

Musso, C. G., Belloso, W. H., Scibona, P., Bellizzi, V., \& Macías Núñez, J. F. (2015). Impact of renal aging on drug therapy. Postgraduate Medicine, 127(6), 623-629. https://doi.org/10.1080/00325481.2015.1063957

Oliveira, M. G., Amorrim, W. W., Oliveira, C. R. B., Coqueiro, H. L., Gusmão, L. C. \& Passos, L. C. (2016). Consenso brasileiro de medicamentos potencialmente inapropriados para idosos. Geriatrics, Gerontology and Aging, 10(4), 168-181. https://cdn.publisher.gn1.link/ggaging.com/pdf/v10n4a02.pdf

Organização Pan-Americana de Saúde (OPAS). (2018). Folha Informativa - Envelhecimento e Saúde. World Health Organisation (pp. 1-6). https://www.paho.org/bra/index.php?option=com_content\&view=article \&id=5661:folha-informativa-envelhecimento-e-saude $\&$ Itemid=820

Palacios, C. R. F., Haugen, E. N., Rasmussen, R. W., \& Thompson, A. M. (2015). Avoidance of polypharmacy and excessive blood pressure control is associated with improved renal function in older patients. Renal Failure, 37(6), 961-965. https://doi.org/10.3109/0886022X.2015.1044399

Reev, E., Ong, M., Wu, A., Jansen, J., Petrovic, M. \& Gnjindic, D. (2017). A systematic review of interventions to deprescribe benzodiazepines and other hypnotics among older people. European Journal Clinical Pharmacology. DOI: 10.1007/s00228-017-2257-8

Rocha, R. S., Silva, L. G. da, Fernandes, M. R., Figueiredo, R. C. de, \& Baldoni, A. de O. (2020). Potenciais interações medicamentosas e medicamentos inapropriados prescritos para usuários da atenção primária à saúde. Revista Brasileira de Farmácia Hospitalar e Serviço de Saúde, 11(2), 1-6. https://doi.org/10.30968/rbfhss.2020.112.0365.RBFHSS

Secora, A., Alexander, C., Ballew, S., Coresh, J., \& Grams, M. (2019). Inappropriate Medication Use in a Community-Based cohort of older adults. Drugs and Aging, 35(8), 735-750. https://doi.org/10.1007/s40266-018-0563-1.KIDNEY

Shi, S., \& Klotz, U. (2011). Age-related changes in pharmacokinetics. Current Drug Metabolism, 12, 601-610. https://doi.org/10.1016/S1543-5946(05)00005$\mathrm{X}$

Silva, J. M., Mendonça, P. P. \& Partata, A. K. (2014). Anti-inflamatórios não-esteróides e suas propriedades gerais. Revista Científica do ITPAC, 7(4). https://assets.unitpac.com.br/arquivos/Revista/74/artigo5.pdf

Stader, F., Siccardi, M., Battegay, M., Kinvig, H., Penny, M. A., \& Marzolini, C. (2019). Repository Describing an Aging Population to Inform Physiologically Based Pharmacokinetic Models Considering Anatomical, Physiological, and Biological Age-Dependent Changes. Clinical Pharmacokinetics, 58(4), 483-501. https://doi.org/10.1007/s40262-018-0709-7

Ungprasert, P., Cheungpasitpom, W, Crowson, C. S. \& Matteson, E. I. (2015). Individual non-steroidal anti-inflamatory drugs andrisko facute kidney injury: a systematic review and meta-analysis of observational studies. European Journal of Internal Medicine, 26(4), 285-91. DOI: 10.1016/j.ejim.2015.03.008

World Health Organization (WHO). (2016). Multisectoral action for a life course approach to healthy ageing: Draft global strategy and plan of action on ageing and health. In Sixty-ninth World Health Assembly (Issue 22 April). https://apps.who.int/gb/ebwha/pdf_files/WHA69/A69_17-en.pdf

World Health Organization (WHO). (2020). Anatomic Therapeutic Chemical (ATC). WHO Collaborating Centr for Drug Statistics Methodology Norwegian Institute of Public Health. Oslo, Norway. https://www.whocc.no/atc_ddd_index/ 